\title{
Constant mean curvature foliations of flat space-times
}

\section{LARS ANDERSSON}

Let $V$ be a maximal globally hyperbolic flat $n+1$-dimensional space-time with compact Cauchy surface of hyperbolic type. We prove that $V$ is globally foliated by constant mean curvature hypersurfaces $M_{\tau}$, with mean curvature $\tau$ taking all values in $(-\infty, 0)$. For $n \geq 3$, define the rescaled volume of $M_{\tau}$ by $\mathcal{H}=|\tau|^{n} \operatorname{Vol}(M, g)$, where $g$ is the induced metric. Then $\mathcal{H} \geq n^{n} \operatorname{Vol}\left(M, g_{0}\right)$ where $g_{0}$ is the hyperbolic metric on $M$ with sectional curvature -1. Equality holds if and only if $(M, g)$ is isometric to $\left(M, g_{0}\right)$.

\section{Introduction.}

\subsection{Scalar curvature and volume.}

Let $M$ be a compact manifold of dimension $n$ of hyperbolic type, with hyperbolic metric $g_{0}$ of sectional curvature -1 . Besson, Courtois and Gallot, see $[15,8,9]$ and references therein, proved that if $g$ is another metric on $M$ with $\operatorname{Ric}_{g} \geq \operatorname{Ric}_{g_{0}}$, then $\operatorname{Vol}(M, g) \geq \operatorname{Vol}\left(M, g_{0}\right)$ with equality if and only if $g$ is isometric to $g_{0}$. In [8] the question is also raised whether a similar conclusion holds under assumptions on the much softer scalar curvature, and it was proved that the scale invariant quantity

$$
\int_{M}\left|R_{g}\right|^{n / 2} \mu_{g}
$$

considered as a function on the space of Riemann metrics, has a local minimum at the hyperbolic structure.

Let $\mathcal{C}$ be the space of conformal classes of metrics on a compact manifold $M$ of dimension $n$. The Yamabe invariant of the conformal class $[g] \in \mathcal{C}$ is

$$
\mu[g]=\inf _{h \in[g]} \operatorname{Vol}(M, h)^{\frac{2-n}{n}} \int_{M} R_{h} \mu_{h} .
$$

\footnotetext{
${ }^{1}$ Supported in part by the Swedish Natural Sciences Research Council (SNSRC), contract no. R-RA 4873-307 and NSF, contract no. DMS 0104402.
} 
The $\sigma$-constant of $M$ is defined as

$$
\sigma(M)=\sup _{[g] \in \mathcal{C}} \mu[g] .
$$

In the case we are considering, $M$ does not admit a metric of nonnegative scalar curvature, and there is a unique representative $h \in[g]$ with $R_{h}=$ $-n(n-1)$. Thus, letting $\mathcal{M}_{-n(n-1)}$ denote the space of metrics with scalar curvature $-n(n-1)$, we have the relations (cf. [21, Lemma 1] for the first equality)

$$
\begin{aligned}
|\sigma(M)|^{n / 2} & =\inf _{g} \int_{M}\left|R_{g}\right|^{n / 2} \mu_{g} \\
& =[n(n-1)]^{n / 2} \inf _{g \in \mathcal{M}_{-n(n-1)}} \operatorname{Vol}(M, g)
\end{aligned}
$$

This gives a dual formulation of the $\sigma$-constant in terms of volume.

There is an ambitious program to solve the geometrization problem in the negative Yamabe class, and as a consequence to understand $\sigma(M)$ in dimension 3, due to M. Anderson, cf. [1] and references therein. The conjecture that one would like to prove concerning the $\sigma$-constant in the case of manifolds of hyperbolic type is the following.

Conjecture 1. Suppose $M$ is a compact 3-dimensional manifold of hyperbolic type. Then the $\sigma$-constant is realized by the hyperbolic metric, i.e.

$$
\sigma(M)=-6 \operatorname{Vol}\left(M, g_{0}\right)^{2 / 3}
$$

where $g_{0}$ is the hyperbolic metric on $M$ with sectional curvature -1 .

See [3] for the general formulation. At present, little is known about $\sigma(M)$ in dimension 3 . The only case where we have some additional information is in dimension 4. Let $M$ be a compact 4-dimensional manifold of hyperbolic type, and let $\mathcal{C}_{\text {flat }}$ denote the space of flat conformal structures. It follows from the Gauss-Bonnet formula that [18, Theorem 8.3]

$$
\inf _{g \in \mathcal{M}_{-12} \cap \mathcal{C}_{\text {flat }}} \operatorname{Vol}(M, g)=\operatorname{Vol}\left(M, g_{0}\right)
$$

The corresponding question appears to be open in dimension 3 .

Using Seiberg-Witten theory, LeBrun has proved the following. Let $M$ be the underlying manifold of a complex surface with even first Betti number. Then [21]

$$
|\sigma(M)|^{2}=32 \pi^{2} c_{1}^{2}(X)
$$

where $X$ is the minimal model of $M$. 


\subsection{The reduced Hamiltonian and volume.}

Let $(g, K)$ be a Riemann metric on $g$ on $M$ and a symmetric 2-tensor $K$. We call $(g, K)$ vacuum data on $M$ if the vacuum Einstein constraint equations

$$
\begin{array}{r}
R_{g}-|K|_{g}^{2}+\left(\operatorname{tr}_{g} K\right)^{2}=0 \\
\nabla_{a} \operatorname{tr}_{g} K-\nabla^{b} K_{a b}=0
\end{array}
$$

are satisfied. Suppose that $\operatorname{tr}_{g} K$ is constant on $M$. Then the quantity

$$
\mathcal{H}=\left|\operatorname{tr}_{g} K\right|^{n} \operatorname{Vol}(M, g)
$$

is scale free, if we give $K$ the dimension of (length), as is natural from the point of view of the Einstein equations, cf. the discussion in [14]. $\mathcal{H}$, which may be thought of as a function $\mathcal{H}(g, K)$ on the space of solutions to the constraint equations (1.2), is the reduced Hamiltonian for the vacuum Einstein equations, in an appropriate gauge. The infimum of $\mathcal{H}$ can be expressed in terms of $\sigma(M)$ in case $M$ does not admit a metric of nonnegative scalar curvature,

$$
\inf _{(g, K)} \frac{n-1}{n} \mathcal{H}(g, K)^{2 / n}=-\sigma(M),
$$

where the infimum is taken over $(g, K)$ solving (1.2). We see that $\sigma(M)$ can be studied in terms of the volume for $h \in \mathcal{M}_{-n(n-1)}$ as well as the Hamiltonian $\mathcal{H}$.

Questions about inf $\mathcal{H}$ are related in spirit to the positive mass problem in general relativity, which concerns the Hamiltonian for the vacuum Einstein equations, in case $M$ is asymptotically Euclidean. In that case, the mass of $M$ is defined by

$$
16 \pi m(g)=\lim _{r \rightarrow \infty} \int_{S_{r}}\left(g_{i j, i}-g_{i i, j}\right) d S^{j} .
$$

The positive mass theorem states that for $\mathrm{AE}$ metrics with nonnegative scalar curvature, $m(g) \geq 0$, with equality if and only if $g$ is flat. In case $M$ is compact, the mass in the above sense is zero.

The $\sigma$-constant conjecture 1 , leads to the following conjecture, which may be though of as an analogoue to positive mass for spatially compact space-times.

Conjecture 2. Suppose $M$ is a compact 3-dimensional manifold of hyperbolic type and let $(g, K)$ be vacuum data on $M$, with constant mean curvature 
$\operatorname{tr} K=\tau$. Then the rescaled volume $\mathcal{H}(g, K)$ satisfies

$$
\mathcal{H}(g, K) \geq 3^{3} \operatorname{Vol}\left(M, g_{0}\right),
$$

where $g_{0}$ is the hyperbolic metric on $M$ with sectional curvature -1 . Equality holds in (1.4) if and only if $g$ is isometric to $\frac{9}{\tau^{2}} g_{0}$, and $K=\frac{\operatorname{tr} K}{3} g$.

The Hamiltonian $\mathcal{H}$ has the property that it is monotone decreasing along the flow of the Einstein evolution equations, in constant mean curvature gauge. This was noticed first by Rendall, and studied in detail by Fischer and Moncrief [14] for dimension 3.

Here we will study the rescaled volume $\mathcal{H}$ in a restricted geometric setting, namely that of flat space-times. Suppose $M$ is compact of hyperbolic type. We call $(g, K)$ flat data on $M$, if

$$
\begin{aligned}
R_{a b}-K_{a c} K_{b}^{c}+\operatorname{tr} K K_{a b} & =0 \\
\nabla_{a} K_{b c}-\nabla_{b} K_{a c} & =0
\end{aligned}
$$

These are just the Gauss and Codazzi equations for a hypersurface in a Lorentzian flat ambient space. We may view the flat data on $M$ with $\operatorname{tr} K=\tau$ for a given $\tau<0$ as a parametrization of the space of maximal globally hyperbolic flat Lorentz structures of topology $M \times \mathbb{R}$. The dimension of the space of flat data, modulo diffeomorphisms, with $\operatorname{tr} K=\tau$, for a fixed $\tau<0$, is equal to the dimension of the Zariski tangent space of the flat conformal structures on $M$.

Theorem 1.1. Let $M$ be a compact manifold of hyperbolic type, of dimension $n \geq 3$. Let $(g, K)$ be flat data on $M$ with $\operatorname{tr} K=\tau$ constant on $M$, and let $\mathcal{H}(g, K)$ be defined by (1.3). Then

$$
\mathcal{H}(g, K) \geq n^{n} \operatorname{Vol}\left(M, g_{0}\right)
$$

where $g_{0}$ is the hyperbolic metric on $M$ with sectional curvature-1. Equality holds in (1.6) if and only if $g$ is isometric to $\frac{n^{2}}{\tau^{2}} g_{0}$ and $K=\frac{\operatorname{tr} K}{n} g$.

The proof of Theorem 1.1 is based on the above-mentioned monotonicity of $\mathcal{H}$, together with two results concerning constant mean curvature foliations of flat space-times. The first, cf. section 3.3, proves the existence of global constant mean curvature foliations.

Theorem 1.2. Let $V$ be a maximal globally hyperbolic flat space-time with compact Cauchy surface $M$ of hyperbolic type. Then $V$ is globally foliated by constant mean curvature hypersurfaces 
For $(g, K)$ induced data on a constant mean curvature hypersurface of mean curvature $\tau<0$, we write $\mathcal{H}(\tau)=\mathcal{H}(g, K)$.

Theorem 1.3. Let $V$ be a maximal globally hyperbolic flat space-time with compact Cauchy surface of hyperbolic type. Then

$$
\lim _{\tau \nearrow 0} \mathcal{H}(\tau)=n^{n} \operatorname{Vol}\left(M, g_{0}\right)
$$

Theorem 1.3 together with the above mentioned monotonicity property proves Theorem 1.1.

Acknowledgements: I am grateful to Misha Gromov, Vince Moncrief, Mike Anderson and Kevin Scannell for helpful discussions. The paper was finished during visits to the Courant institute, New York and Institute Elie Cartan, Nancy. The hospitality and support during these visits is gratefully acknowledged.

\section{Preliminaries.}

A $(G, X)$ structure on $V$ is a marked locally homogenous structure, modelled on a homogenous space $X=G / H$. A $(G, X)$-structure on $V$ gives by a standard construction a $(G, X)$-structure on the universal cover $\tilde{V}$, a developing map $\mathcal{D}: \tilde{V} \rightarrow X$ and a holonomy homomorphism $\rho: \pi_{1} V \rightarrow G$ satisfying the equivariance condition $\mathcal{D}(\gamma \cdot x)=\rho(\gamma) \cdot \mathcal{D}(x)$ for all $\gamma \in \pi_{1} V$, $x \in \tilde{V}$.

The $n+1$-dimensional Minkowski space $\mathbb{R}_{1}^{n+1}$, is $\mathbb{R}^{n+1}$ with the flat Lorentz metric $-d t^{2}+\left(d x^{1}\right)^{2}+\cdots+\left(d x^{n}\right)^{2}$. We will use coordinates $t, x^{1}, \ldots x^{n}$ or $x^{0}, x^{1}, x^{n}$ on $\mathbb{R}_{1}^{n+1}$, and write $|x|=\left(\sum_{i=1}^{n}\left(x^{i}\right)^{2}\right)^{1 / 2}$. The group of linear isometries of $\mathbb{R}_{1}^{n+1}$ is the Lorentz group $\mathrm{O}(n, 1)$, and the group of isometries of $\mathbb{R}_{1}^{n+1}$ is the semidirect product $\operatorname{ISO}(n, 1)=\mathrm{O}(n, 1) \ltimes \mathbb{R}^{n+1}$ of the Lorentz group with the group of translations of $\mathbb{R}^{n+1}$. We will denote by $\mathrm{O}(n, 1)_{+}$the subgroup of time orientation preserving Lorentz transformations, by $\mathrm{SO}(n, 1)$ the group of Lorentz transformations of determinant 1 , and by $\mathrm{SO}(n, 1)_{0}$ the connected component of the identity in $\mathrm{O}(n, 1)$. Similarly $\operatorname{ISO}(n, 1)_{0}$ denotes the connected component of the identity in ISO $(n, 1)$. The frame bundle of $\mathbb{R}_{1}^{n+1}$ has a natural $\mathrm{SO}(n, 1)_{0}$ reduction.

Examples of $(G, X)$-structures in dimension $n$ are hyperbolic structures, with $\left(G=S O(n, 1), X=\mathbb{H}^{n}\right)$, flat conformal structures, with $(G=\operatorname{SO}(n+$ $\left.1,1), X=S^{n}\right)$, and flat Lorentz structures, with $\left(G=\operatorname{ISO}(n, 1), X=\mathbb{R}_{1}^{n+1}\right)$. 


\subsection{Deformation spaces.}

The space of $(G, X)$ structures on $V$ is isomorphic to the deformation space of holonomy representations $\rho: \pi_{1} V \rightarrow G$. The formal (Zariski) tangent space of the deformation space at $\rho$ is, letting $\Gamma=\rho \pi_{1} V$, given by the cohomology $H^{1}\left(\Gamma, \mathfrak{g}_{\mathrm{Ad}}\right)$, where $\mathfrak{g}_{A d}$ denotes the adjoint action of $G$ on $\mathfrak{g}=\operatorname{Lie}(G)$. In general, the deformation space is not a manifold, for example the space of flat conformal structures may have singularities at the hyperbolic strucure on a compact manifold of hyperbolic type in dimension $n \geq 4$ [18]. Obstructions to integrating a direction in the Zariski tangent space can be computed in terms of Massey products [17].

Let $\mathfrak{i s o}(n, 1)=\operatorname{Lie}(\operatorname{ISO}(n, 1)), \mathfrak{s o}(n+1,1)=\operatorname{Lie}(\operatorname{SO}(n+1,1))$. Note that $\mathfrak{s o}(n+1,1)=\mathfrak{s o}(n, 1) \oplus \mathfrak{m}, \mathfrak{m}=\mathbb{R}^{n+1}$ as a vector space, and $\mathfrak{i s o}(n, 1)=$ $\mathfrak{s o}(n, 1) \oplus \mathbb{R}^{n+1}$, where $\mathbb{R}^{n+1}$ has the trivial Lie algebra structure. The adjoint action of $\mathrm{SO}(n, 1) \subset \mathrm{SO}(n+1,1)$ on $\mathfrak{s o}(n+1,1)$ induces an action on $M$ which is equivalent to the vector action of $\mathrm{SO}(n, 1)$ on $\mathbb{R}^{n+1}$. We let $\mathbb{R}_{\text {vec }}^{n+1}$ denote the vector representation of $\mathrm{SO}(n, 1)$ on $\mathbb{R}^{n+1}$.

Let $\Gamma$ be the holonomy representation for a hyperbolic structure on $M$. Then

$$
\begin{aligned}
H^{1}\left(\Gamma, \mathfrak{s o}(n+1,1)_{\mathrm{Ad}}\right) & =H^{1}\left(\Gamma, \mathfrak{s o}(n, 1)_{\mathrm{Ad}}\right) \oplus H^{1}\left(\Gamma, \mathfrak{m}_{\mathrm{Ad}}\right), \\
H^{1}\left(\Gamma, \mathfrak{i s o}(n, 1)_{\mathrm{Ad}}\right) & =H^{1}\left(\Gamma, \mathfrak{s o}(n, 1)_{\mathrm{Ad}}\right) \oplus H^{1}\left(\Gamma, \mathbb{R}_{\text {vec }}^{n+1}\right) .
\end{aligned}
$$

For $n \geq 3, H^{1}\left(\Gamma, \mathfrak{s o}(n, 1)_{\mathrm{Ad}}\right)=\{0\}$, by Calabi-Weil rigidity, so $H^{1}\left(\Gamma, \mathbb{R}_{\mathrm{vec}}^{n+1}\right)$ is the Zariski tangent space for both flat conformal structures and flat Lorentz structures. For $n=2$ on the other hand, the adjoint representation of $\mathrm{SO}(2,1)$ on $\mathfrak{s o}(2,1)$ is equivalent to the vector representation on $\mathbb{R}^{3}$, so

$$
H^{1}\left(\Gamma, \mathfrak{i s o}(2,1)_{\mathrm{Ad}}\right)=H^{1}\left(\Gamma, \mathbb{R}_{\mathrm{vec}}^{3}\right) \oplus H^{1}\left(\Gamma, \mathbb{R}_{\mathrm{vec}}^{3}\right) .
$$

Further, in case $=2, \operatorname{dim} H^{1}\left(\Gamma, \mathbb{R}_{\mathrm{vec}}^{3}\right)=6 \operatorname{genus}(M)-6$.

Let $d^{\nabla}$ be defined on symmetric 2-tensors by $d^{\nabla} h_{a b c}=\nabla_{c} h_{a b}-\nabla_{b} h_{a c}$. Thus equation (1.5b) can be written as $d^{\nabla} K=0$. Tensors satisfying $d^{\nabla} h=0$ are called Codazzi tensors. The following proposition shows the relation between trace-free Codazzi tensors and deformations.

Proposition 2.1 (Lafontaine [20]). Let $M$ be a compact hyperbolic manifold of dimension $n \geq 2$, with holonomy representation $\Gamma \subset \mathrm{SO}(n, 1)$. Then

$$
\mathbb{H}^{1}\left(\Gamma, \mathbb{R}_{\text {vec }}^{n+1}\right) \cong \operatorname{ker} d^{\nabla} \cap \operatorname{ker} \operatorname{tr} .
$$


In case $n=2$, the trace-free Codazzi tensors are precisely the holomorphic quadratic differentials. Thus, for $n \geq 3$, the deformation space of flat Lorentz structures has the same dimension as the space of trace-free Codazzi tensors on $M$, while for $n=2$, the space of flat Lorentz structures has the dimension twice that of the Teichmuller space of $M$.

Let $h \in \operatorname{ker} d^{\nabla} \cap$ ker tr. A cocycle $\alpha \in Z^{1}\left(\Gamma, \mathbb{R}_{\text {vec }}^{n+1}\right)$ corresponding to $h$ may be constructed as follows [20]. Let $\tilde{h}$ be the lift of $h$ to the universal cover $\mathbb{H}^{n}$ of $\left(M, g_{0}\right)$. Then $\tilde{h}=\nabla^{2} f-f g_{0}$, for some $f \in C^{\infty}$, cf. the note by D. Ferus in [10]. Then $\alpha(\gamma)=f \circ \gamma-f$ is a cocycle. The function $f$ is unique up to addition by a function $\beta$ which is the restriction to $\mathbb{H}^{n} \subset \mathbb{R}^{n+1}$ of a linear function. If $f$ is replaced by $f_{1}=f+\beta$, then $\alpha$ is replaced by $\alpha_{1}(\gamma)=\alpha(\gamma)+\beta \circ \gamma-\beta$, which represents the same class in $H^{1}\left(\Gamma, \mathbb{R}_{\mathrm{vec}}^{n+1}\right)$ as $\alpha$, since $\beta \circ \gamma-\beta$ is exact. One shows that $\alpha$ is nontrivial if $h \neq 0$.

Let $\left(M, g_{0}\right)$ be compact hyperbolic with metric $g_{0}$ of sectional curvature -1. Then the Lorentz cone $(V, \eta)$ over $M, V=M \times(0, \infty), \eta=-d \rho^{2}+\rho^{2} g_{0}$, is a flat, globally hyperbolic space-time, cf. Proposition 2.2. If $M$ has a two-sided nonsingular totally geodesic hypersurface, then $H^{1}\left(\Gamma, \mathbb{R}_{\text {vec }}^{n+1}\right) \neq 0$, cf. [18]. Let $\Sigma \subset M$ be such a totally geodesic hypersurface, and let $h$ be the induced metric on $M$. Then the Lorentz cone $\left(V^{\prime}, \eta^{\prime}\right)$ over $(\Sigma, h)$, $V^{\prime}=\Sigma \times(0, \infty), \eta^{\prime}=-d \rho^{2}+\rho^{2} h$, is totally geodesic in $(V, \eta)$. It follows that replacing $V^{\prime}$ by a Kasner type space-time $W=V^{\prime} \times(0, R)$, with line element

$$
-d \rho^{2}+\rho^{2} h+d r^{2}
$$

gives a flat space-time which is a deformation of $(V, \eta)$.

In dimension 3, it is still an open question whether or not the space of flat conformal structures is a manifold. It has been conjectured by Kapovich [19], that the space of flat conformal structures on a compact hyperbolic manifold is smooth in dimension 3.

In the case of flat Lorentz structures, on the other hand, the situation is simplified due to the fact that the Lie algebra structure on $\mathbb{R}^{n+1}$ is trivial. It follows from this that the obstructions are all trivial and that the deformation space of flat Lorentz structures is smooth in all dimensions.

\subsection{Space-times.}

We will use both terms "Lorentz manifold" and "space-time" interchangeably. A space-time $(V, \eta)$ is said to be vacuum if it is Ricci flat.

A space-like hypersurface $M$ in a Lorentz manifold $V$ is said to be a Cauchy surface if $V$ is the domain of dependence of $M$, i.e. if every inex- 
tendible causal curve in $V$ intersects $M$. If $V$ has a Cauchy surface, we say that $V$ is globally hyperbolic. Given a vacuum space-time $V$, a vacuum extension of $V$ is a vacuum space-time $V^{\prime}$ of the same dimension as $V$, together with an isometric imbedding $i: V \rightarrow V^{\prime}$, so that $i(V)$ is a proper subset of $V^{\prime}$. Inclusion induces a partial order on the class of space-times and also on the class of globally hyperbolic vacuum space-times, so that it is natural to talk about the maximal globally hyperbolic vacuum extension of a space-time $V$. These concepts apply directly also to the class of globally hyperbolic flat space-times.

If $(M, g, K)$ is a space-like hypersurface in a vacuum space-time $V$, with induced Riemann metric $g$ and second fundamental form $K$, then $(g, K)$ is a vacuum data set. Similarly, in case $V$ is flat, we say that the induced data $(g, K)$ is a flat data set. See section 1.2. If $V$ is a vacuum (flat) space-time, such that $(M, g, K)$ embeds in $V$ as a Cauchy hypersurface, then $V$ is called a vacuum (flat) globally hyperbolic extension of $(M, g, K)$.

In the class of vacuum space-times, the maximal vacuum globally hyperbolic extension is unique up to isometry, as a consequence of uniqueness for the Cauchy problem for the Einstein equations in space-harmonic coordinates. The following Lemma shows that this holds also in the flat case.

Lemma 2.1. 1. Every flat globally hyperbolic space-time has a unique maximal globally hyperbolic flat extension.

2. Let $(M, g, K)$ be a flat data set. Then there is a maximal globally hyperbolic flat extension $V$ which is unique up to isometry. In particular, if $\phi: M \rightarrow M$ is a diffeomorphism, and $g^{\prime}=\phi^{*} g, K^{\prime}=\phi^{*} K$, then the maximal globally hyperbolic flat extensions of $(M, g, K)$ and $\left(M, g^{\prime}, K^{\prime}\right)$ are isometric.

Proof Let $V$ be a flat, globally hyperbolic space-time. Since $V$ is flat it is also vacuum. By a theorem of Choquet-Bruhat and Geroch [13], every vacuum globally hyperbolic space-time has a unique maximal globally hyperbolic vacuum extension $V^{\prime}$.

Let $W$ denote the Weyl tensor of $V^{\prime}$. A calculation shows $\square W=F(W)$, where $\square$ is the covariant wave operator on $V^{\prime}$ and $F$ is a quadratic expression in $W$. Uniqueness for the Cauchy problem shows that if $W$ is vanishes on $V$, it vanishes on $V^{\prime}$, and consequently $V^{\prime}$ is flat. This proves part 1 . The argument for part 2 is analogous.

For brevity we will use the acronym MGHF for maximally globally hyperbolic flat. In the following we will let $V$ be a MGHF space-time of dimension 
$n+1, n \geq 2$, with compact Cauchy surface $M$ of hyperbolic type, i.e. $M$ admits a hyperbolic structure.

\subsection{Flat space-times.}

The presentation in this section follows to a large extent the work in the unpublished preprint of G. Mess [23], which dealt with 2+1-dimensional constant curvature space-times. In particular, most of the proofs in the rest of this section are relatively straightforward generalizations of the proofs of the analogous results in [23], and as the concluding paragraph of [23] indicates, at least some of these generalizations were known to G. Mess.

A MGHF space-time $V$ with Cauchy surface $M$ has topology $M \times R$, and consequently, $\pi_{1} V \cong \pi_{1} M$. A flat Lorentz metric on an $n+1$ dimensional, smooth manifold $V$ defines a (ISO $\left.(n, 1), \mathbb{R}_{1}^{n+1}\right)$-structure on $V$. Letting $\Gamma=$ $\rho\left(\pi_{1} V\right)$ be the holonomy representation we can also write $\Gamma=\rho\left(\pi_{1} M\right)$. The universal cover $\tilde{V}$ is again globally hyperbolic and from this it follows that $\Gamma \subset \operatorname{ISO}(n, 1)_{0}$, i.e. $V$ is orthochronous. The development $\mathcal{D}(\tilde{V})$ is a globally hyperbolic subset of $\mathbb{R}_{1}^{n+1}, \Gamma$ acts properly discontinuously on $\mathcal{D}(\tilde{V})$ and hence on the restriction of the $\mathrm{SO}(n, 1)_{0}$ bundle over $\mathbb{R}_{1}^{n+1}$ to $\mathcal{D}(\tilde{V})$. It follows that $\Gamma$ is discrete in $\operatorname{ISO}(n, 1)_{0}$.

The following Lemma is a straightforward generalization of [23, Proposition 4] and [27, Lemma 2.1]. We include the proof for completeness.

Lemma 2.2. The image of $\mathcal{D}: \tilde{M} \rightarrow \mathbb{R}_{1}^{n+1}$ embeds $M$ as a space-like graph over $\mathbb{E}^{n}=\left\{(t, x) \in \mathbb{R}_{1}^{n+1}: t=0\right\}$, and $t$ is a proper function on $\tilde{M}$.

Proof The natural projection $\mathbb{R}_{1}^{n+1} \rightarrow \mathbb{E}^{n}$ restricts to a local diffeomorphism $P: \mathcal{D}(\tilde{M}) \rightarrow \mathbb{E}^{n}$, since $D(\tilde{M})$ is everywhere space-like. As $M$ is compact and strictly space-like, the induced Riemann metric on $\mathcal{D}(\tilde{M})$ is complete. Further, the pullback to $\mathcal{D}(\tilde{M})$ of the metric on $\mathbb{E}^{n}$ is pointwise larger than the induced metric on $\mathcal{D}(\tilde{M})$ and is therefore complete. This shows that $P$ is a covering map and hence a global diffeomorphism. Since $\tilde{M}$ is connected, it follows that $\mathcal{D}(\tilde{M})$ is a graph.

An element $\gamma \in \Gamma$ is of the form $x \mapsto A x+a$. Let $L: \operatorname{ISO}(n, 1)_{0} \rightarrow \operatorname{SO}(n, 1)_{0}$ be the projection on the linear part, $L(A, a)=A$, and let $f: \pi_{1} M \rightarrow$ $\mathrm{SO}(n, 1)_{0}$ be the homomorphism $f=L \rho$. Clearly $\operatorname{ker} L=\mathbb{R}^{n+1}$. The short exact sequence

$$
1 \rightarrow \mathbb{R}_{1}^{n+1} \rightarrow \mathrm{ISO}(n, 1) \stackrel{L}{\rightarrow} \mathrm{O}(n, 1) \rightarrow 1
$$


leads to a short exact sequence

$$
1 \rightarrow T(\Gamma) \rightarrow \Gamma \stackrel{L}{\rightarrow} L(\Gamma) \rightarrow 1,
$$

where $T(\Gamma)$ is the kernel of $L$ restricted to $\Gamma$.

For $n=2$, the following Lemma is due to Mess [23, Proposition 1]. The case $n=3$ follows from [27].

Lemma 2.3. 1. $T(\Gamma)=0$ and $L: \Gamma \rightarrow L(\Gamma)$ is an isomorphism.

2. $L(\Gamma)$ is a discrete cocompact subgroup of $\mathrm{SO}(n, 1)_{0}$. In case $n \geq 3, f$ is unique up to conjugation.

Proof Since the image under the development map of $\tilde{M}$ is space-like, $T(\Gamma)$ must consist of space-like vectors. Since $\Gamma$ is discrete, and $T(\Gamma)$ space-like, it follows $T(\Gamma)=Z^{k}$, some $k \leq n$. By assumption, $\Gamma=\pi_{1} M$ and $M$ is of hyperbolic type. This means $\Gamma$ has no normal subgroups $Z^{k}$ and hence $T(\Gamma)=0$. This proves part 1 .

For part 2, we use an argument due to Scannell [27], [28]. Suppose for a contradiction, $L(\Gamma)$ is indiscrete and let $\overline{L(\Gamma)}$ denote the closure of $L(\Gamma)$ in $\mathrm{SO}(n, 1)$. By [25, Theorem 8.24], the identity component ${\overline{L(\Gamma)_{0}}}_{\text {is }}$ solvable. By [26, Theorem 5.5.10], $\overline{L(\Gamma)}_{0}$ is elementary, and it follows from the classification of elementary subgroups in $[26, \S 5.5]$ that the set of points $F$ on the sphere at infinity $\partial \mathbb{H}^{n}$, left invariant by $\overline{L(\Gamma)_{0}}$ must consist of one or two points. The stabilizer in $\mathrm{SO}(n, 1)_{0}$ of a point at infinity is isomorphic to the group $\operatorname{Sim}^{+}\left(\mathbb{R}^{n-1}\right)$ or orientation preserving similarity transformations of $\mathbb{R}^{n-1}$, which is a solvable group. Since $L(\Gamma)$ normalizes $\overline{L(\Gamma)_{0}}$, it leaves $F$ invariant and is therefore solvable, a contradiction to the assumption that $\Gamma=\pi_{1} M$ for $M$ of hyperbolic type. The rigidity statement follows from Mostow rigidity.

Proposition 2.2. Given $f: \pi_{1} M \rightarrow \mathrm{SO}(n, 1)_{0}$, discrete and faithful, there is a MGHF space-time $V=M \times(0, \infty)$ with holonomy $\rho: \pi_{1} V \rightarrow \operatorname{ISO}(n, 1)$ equal to $f$.

Proof Let $\Gamma=f \pi_{1} M$. Then $\Gamma$ acts discontinously on $I^{+}(\{0\})$, the interior of future light cone at $0 \in \mathbb{R}_{1}^{n+1}$. The quotient $V=I^{+}(\{0\}) / \Gamma$ is the required space-time. $V$ is globally hyperbolic since $I^{+}(\{0\})$ is.

The flat space-time $V$ is the Lorentz cone over the compact hyperbolic manifold $M$. We will refer to such space-times as hyperbolic cone spacetimes. The metric on a hyperbolic cone space-time $V=(0, \infty) \times M$ has the 
warped product form

$$
\eta=-d \rho^{2}+\rho^{2} g_{0}
$$

where $g_{0}$ is the hyperbolic metric on $M=\mathbb{H}^{n} / \Gamma$. Here $\rho$ corresponds to the Lorentz distance to 0 in $\mathbb{R}_{1}^{n+1}$. The hypersurface $\rho=s$ has constant mean curvature $\tau=-n / s$, so that $\{\rho=1\}$ is $\mathbb{H}^{n}$.

\subsection{Existence of strictly convex Cauchy surfaces.}

For $\gamma \in \pi_{1} M, \rho(\gamma) x=f(\gamma) x+t_{\gamma}$, where $t: \pi_{1} M \rightarrow \mathbb{R}^{n+1}, \gamma \mapsto t_{\gamma}$ is a 1-cocycle, i.e. $t_{\alpha \beta}=t_{\alpha}+f(\alpha) t_{\beta}$.

The quotient space $H^{1}\left(f\left(\pi_{1} M\right), \mathbb{R}^{n+1}\right)$ of the cocycles by the coboundaries, corresponds to the space of conjugacy classes of representations $\rho: \pi_{1} M \rightarrow \operatorname{ISO}(n, 1)_{0}$, such that $L \rho=f$. We fix a linear identification $u \mapsto t(u)$, of $H^{1}\left(f\left(\pi_{1} M\right), \mathbb{R}^{n+1}\right)$ with a subspace of the space of cocycles $Z^{1}\left(f\left(\pi_{1} M\right), \mathbb{R}^{n+1}\right)$.

Proposition 2.3 (Analogue of [23, Proposition 3]). Given $M$ compact of hyperbolic type and $f: \pi_{1} M \rightarrow \mathrm{SO}(n, 1)_{0}$, the holonomy representation for a hyperbolic structure on $M$, let $V$ be the hyperbolic cone space-time with holonomy $H=f \pi_{1} M$. Then for any bounded neighborhood $U$ of $0 \in H^{1}\left(H, \mathbb{R}^{n+1}\right)$, there exists $C>0$ so that, letting

$$
V_{C}=\left\{(t, x):-t^{2}+|x|^{2} \leq-C, \quad t>0\right\} / H
$$

there exists a family of flat Lorentz metrics on $V_{C}$, parametrized by $U$, so that the space-time $V_{C}(U)$, corresponding to $u \in U$ has holonomy $\rho: \pi_{1} M \rightarrow$ $\operatorname{ISO}(n, 1)_{0}$, sich that $L \rho=f$, and $\rho(\gamma) x=f(\gamma) x+t_{\gamma}(u)$, where $t_{\gamma}(u)$ is a cocycle representing $u \in H^{1}\left(\pi_{1} M, \mathbb{R}^{n+1}\right)$. We say that $V_{C}(u)$ represents $u$. Moreover the space-times $V_{C}(u)$ are future complete and $M$ is locally strictly convex in each $V_{C}(u)$.

Proof Consider the space-time

$$
V^{\prime}=\left\{(x, t), x \in \mathbb{R}^{n}, t>0, \quad-\frac{1}{2} \geq-t^{2}+|x|^{2} \geq-2\right\} / f \pi_{1} M .
$$

Due to the fact that $H^{1}\left(f\left(\pi_{1} M\right)\right.$, iso $\left.(n, 1)\right)=H^{1}\left(f\left(\pi_{1} M\right), \mathbb{R}^{n+1}\right)$, where $\mathbb{R}^{n+1}$ has the trivial Lie algebra structure (cf. the discussion above), the obstructions to integrating a ray in $Z^{1}\left(f\left(\pi_{1}(M), \mathbb{R}^{n+1}\right)\right.$ vanish, see [17] for background, and hence by [7], each ray corresponds to a curve in the deformation space of flat Lorentz structures. It follows from this 
that there is a neighborhood $U_{0}$ of 0 in $H^{1}\left(\pi_{1} M, \mathbb{R}^{n+1}\right)$, and a family of $\left.\operatorname{ISO}(n, 1)_{0}, \mathbb{R}^{n+1}\right)$ structures on $V^{\prime}$, parametrized by $U_{0}$, such that the holonomy $\rho: \pi_{1} V^{\prime}(u) \rightarrow \operatorname{ISO}(n, 1)_{0}$ represents $u$. This can also be seen directly by considering the parametrized development map $\mathcal{D}: \tilde{M}^{\prime} \times U_{0} \rightarrow \mathbb{R}^{n+1}$, defined by

$$
\mathcal{D}(\gamma \cdot x, v)=f(\gamma) \cdot x+t_{\gamma}(v)
$$

Choose $\lambda>0$ so that $U \subset \lambda U_{0}$ and let

$$
V^{\prime \prime}=\lambda \cdot V^{\prime}=\left\{(t, x) \in \mathbb{R}^{n+1}, t>0, \quad-\frac{\lambda^{2}}{2} \geq-t^{2}+|x|^{2} \geq-2 \lambda^{2}\right\} / \pi_{1} M .
$$

If we define $\mathcal{D}^{\prime}: \tilde{V}^{\prime \prime} \times \lambda \cdot U_{0} \rightarrow \mathbb{R}^{n+1}$ by

$$
\mathcal{D}^{\prime}(\lambda \cdot x, \lambda \cdot u)=\lambda \cdot(\mathcal{D}(x, u)),
$$

then

$$
\begin{aligned}
\mathcal{D}^{\prime}(\gamma x, u) & =\lambda \mathcal{D}(\gamma x / \lambda, u / \lambda) \\
& =\lambda\left(f(\gamma) x / \lambda+t_{\gamma}(u / \lambda)\right) \\
& =f(\gamma) x+t_{\gamma}(u) .
\end{aligned}
$$

Thus $\mathcal{D}^{\prime}$ is the parametrized developing map of a family of flat Lorentz structures. We observe that if $U_{0}$ is chosen sufficiently small, then with

$$
N=\left\{-t^{2}+|x|^{2}=-1\right\} / f \pi_{1} M,
$$

$N$ remains locally strictly convex in the Lorentz structure determined by any $u \in U_{0}$. It follows that $\mathcal{D}(\tilde{N}, u)$ is a strictly convex complete space-like hypersurface.

The following corollary, which is an immediate consequence of the proof of Proposition 2.3, provides barriers which will be used to control the limit of constant mean curvature hypersurfaces in the expanding direction.

Corollary 2.4. Let $\varepsilon>0$ be given. Then, in the situation of Proposition 2.3 , by choosing $U_{0}$ sufficiently small, the hypersurfaces $N_{s}, 3 / 4 \leq s \leq 5 / 4$, defined by

$$
N_{s}=\left\{-t^{2}+|x|^{2}=-s^{2}\right\}
$$

are strictly locally convex in the Lorentz structure corresponding to $u \in U_{0}$, and the mean curvature $\tau_{s, u}$ of $N_{s}$ satisfies

$$
\left|\tau_{s, u}+n / s\right|<\varepsilon
$$

Further $N_{s_{1}}$ is in the future of $N_{s_{2}}$ for $s_{1} \geq s_{2}$. 
Definition 1 (Standard space-time). A flat globally hyperbolic spacetime $V$ with compact hypersurface $M$ of hyperbolic type is a standard space-time if $V$ is the quotient of the future in $\mathbb{R}_{1}^{n+1}$ of a complete spacelike strictly convex hypersurface $\tilde{M}$ by a group of Lorentz isometries acting cocompactly on $\tilde{M}$.

Given $V$ with compact Cauchy surface $M$, we may assume $t \nearrow \infty$ on $\tilde{M}$, after a time orientation. We say $M$ is future oriented.

Proposition 2.5 (Analogue of [23, Proposition 5]). Suppose $V$ is a flat space-time with compact Cauchy surface $M$ of hyperbolic type. There exists a standard space-time $V^{\prime}$ containing a future directed strictly convex surface $M^{\prime}$ of the same topology and so that $M$ and $M^{\prime}$ have the same holonomy representation. Furthermore, $\tilde{M}^{\prime}$ can be chosen to lie in the future of $\tilde{M}$.

Proof By Lemma 2.3 and Proposition 2.3, there is a standard space-time $V^{\prime}$ containing a locally strictly convex hypersurface $M^{\prime}$ such that, after fixing some identification between $\pi_{1} M$ and $\pi_{1} M^{\prime}$, the holonomy homomorphisms from $\pi_{1} M$ and $\pi_{1} M^{\prime}$ are equal. Replace $M^{\prime}$ by $M^{\prime}(K)$, the hypersurface of points which lie on the future pointing normals to $M^{\prime}$ at proper time $K . M$ is compact, so there exists $K$ sufficiently large so that for each $p \in \tilde{M}$, some point of $\tilde{M}^{\prime}(K)$ lies in the future of $p$. Then $\tilde{M}^{\prime}(K)$ lies entirely in the future component of $\mathbb{R}_{1}^{n+1} \backslash \tilde{M}$, because no time-like line joins two points in $\tilde{M}^{\prime}$.

Proposition 2.6 (Analogue of [23, Proposition 6]). Let $V$ be a flat space-time with compact Cauchy surface $M$. Then there is a flat spacetime $V^{\prime \prime}$, containing a neighborhood of $S$ in $V$, and containing a standard space-time $V^{\prime}$.

Proof Let $\tilde{M}^{\prime}$ be a space-like strictly convex hypersurface in $V^{\prime}$ so that for each $m \in \tilde{M}$, some point of $\tilde{M}^{\prime}$ is in the future of $m$ (i.e. $\tilde{M}$ is in the past domain of dependence of $\tilde{M}^{\prime}$ ) and $\tilde{M}^{\prime}$ is also invariant under the holomony represenation $\rho\left(\pi_{1} M\right)$ of $V$. It follows from Proposition 2.5 that there exists such $\tilde{M}^{\prime}$. $\tilde{M}^{\prime}$ together with its future is the development of our standard space-time $V^{\prime}$. To construct $V^{\prime \prime}$, it is sufficient to show that $\rho\left(\pi_{1} M\right)$ acts properly discontinuously on the region $R$ between the disjoint space-like hypersurfaces $\tilde{M}$ and $\tilde{M}^{\prime}$. We then adjoin $R / \rho\left(\pi_{1} M\right)$ to $M^{\prime}$ and thicken the resulting manifold slightly to the past.

Define a map $h: \tilde{M} \times[0,1] \rightarrow R$ by $(m, u) \rightarrow(1-u) m+u \varphi(m)$ where $\varphi(m)$ is the intersection of the future pointing normal to $\tilde{M}$ at $m$ with $\tilde{M}^{\prime}$. 
The map $h$ is proper: a compact subset $K$ of $R$ lies in a region $t \leq C$ for some $C$, so $h^{-1} K \subset \tilde{M}$ is contained in the set $A=\{m \in \tilde{M}: t \leq \bar{C}\}$. By Lemma $2.2, A$ is compact.

To see that $\rho\left(\pi_{1} M\right)$ acts properly discontinously on $R$, let $K \subset R$ be compact. Then for all but finitely many $g \in \rho\left(\pi_{1} M\right.$,

$$
\left[g \cdot h^{-1}(K)\right] \cap h^{-1}(K)=\emptyset=h^{-1}(g K) \cap h^{-1}(K),
$$

so $g K \cap K=\emptyset . \rho\left(\pi_{1} M\right.$ is torsion free so $R$ covers $R / \rho\left(\pi_{1} S\right)$.

The following Theorem is a direct consequence of Proposition 2.6.

Theorem 2.7. Let $V$ be an MGHF space-time with compact Cauchy surface $M$ of hyperbolic type. Then $V$ contains a locally strictly convex Cauchy surface to the future of $M$.

\section{Constant mean curvature foliations.}

\subsection{Existence of a CMC hypersurface.}

A hypersurface $M$ in a space-time $V$ is said to have constant mean curvature (CMC) is $\operatorname{tr} K$ is constant on $M$. CMC hypersurfaces satisfy a space-time maximum principle, and existence of CMC hypersurfaces can be proved using barriers. We use the existence of a strictly convex hypersurface to construct barriers.

Theorem 3.1. Let $V$ be a MGHF space-time with compact Cauchy surface $M$ of hyperbolic type. Then $V$ contains a CMC Cauchy surface to the future of $M$.

Proof By Theorem 2.7, $V$ contains a locally strictly convex Cauchy surface $M^{\prime}$ to the future of $M$. Let $K_{b}^{a}(t)$ be the mixed form of the second fundamental form of the Gauss foliation $\left\{S_{t}\right\}_{t=0}^{\infty}$, to the future (the expanding direction) of $M^{\prime}$, with the sign convention

$$
\partial_{t} g_{a b}=-2 K_{a b},
$$

where $g_{a b}$ is the induced Riemann metric on $S_{t} . K^{a}{ }_{b}$ is the shape operator of the Gauss foliation and satisfies the Riccati equation

$$
\partial_{t} K_{b}^{a}=K_{c}^{a} K_{b}^{c}
$$


By diagonalizing this is seen to have the solution

$$
K(t)=\left(K^{-1}(0)-t\right)^{-1},
$$

and it follows that $\operatorname{tr} K(t)$ is strictly increasing with $t$, and $\operatorname{tr} K(t) \nearrow 0$ as $t \rightarrow \infty$. Since $\operatorname{tr} K(0)<C<0$, it follows that there is a $t_{0}$ so that $M^{\prime}, S_{t_{0}}$ form barriers for the CMC problem and by [16], there is a CMC Cauchy surface $M^{\prime \prime}$ in $V$ to the future of $M$. The mean curvature $\tau$ of $M^{\prime \prime}$ can be choosen to have any value in the open interval $(\max \operatorname{tr} K(0), 0)$.

\subsection{CMC Cauchy surfaces are strictly convex.}

By results of Treibergs and Choi, entire CMC hypersurfaces in $\mathbb{R}_{1}^{n+1}$ have the property that the second fundamental form $K$ is semidefinite, and if $K$ has a zero eigenvalue at some point, then the CMC hypersurface splits. We use this to prove the following Proposition.

Proposition 3.2. Let $V$ be a MGHF space-time with compact CMC Cauchy surface $M$ of hyperbolic type. Then $M$ is strictly locally convex.

Proof We may assume the mean curvature $\tau$ of $M$ satisfies $\tau<0$. Consider $\tilde{M}$, the development of $M$ in $\mathbb{R}^{n+1}$. By [29], the second fundamental form $K$ of $\tilde{M}$ is negative semi-definite. We must prove that $K$ is negative definite. Suppose for a contradiction, there exists $m \in \tilde{M}$ such that $K$ has a zero eigenvalue. Then by [12], $\tilde{M}$ splits metrically, $\tilde{M}=\tilde{M}^{n-k} \times \mathbb{R}^{k}$ for some $k$, $1 \leq k \leq n$, where $\tilde{M}^{n-k}$ has negative definite second fundamental form and the same mean curvature as $\tilde{M}$. In particular, the rank of $K$ is constant.

The tangent spaces split $T_{m} \tilde{M}=E_{1, m} \oplus E_{2, m}$, where $E_{1, m}, E_{2, m}$ are of dimension $n-k$ and $k$, respectively. If we let $P$ denote the orthogonal projection on $\mathbb{R}^{k}, E_{2, m}=P T_{m} \tilde{M}$. According to this splitting, $K=K_{1} \oplus 0$, where $K_{1}<0$.

Let $\rho: \pi_{1} M \rightarrow \operatorname{ISO}(n, 1)_{0}$ be the holonomy representation of $V$. For $\gamma \in \pi_{1} M, \rho(\gamma)$ is an affine isometry of $\mathbb{R}_{1}^{n+1}, \rho(\gamma) m=f(\gamma) m+t_{\gamma}$, where $f=L \rho$.

Now we show that $f(\gamma)$ commutes with $P$, i.e. $f(\gamma) E_{1, m}=f(\gamma) E_{1, \rho(\gamma) m}$, $f(\gamma) E_{2, m}=E_{2, \rho(\gamma) m}$. To see this, use the fact that $\rho(\gamma)^{*} K=K$ by construction. This implies that if we choose $v \in E_{2, m}, w \in T_{m} \tilde{M}$,

$$
0=\rho(\gamma)^{*} K_{m}(v, w)=K_{\rho(\gamma) m}\left(\rho(\gamma)_{*} v, \rho(\gamma)_{*} w\right)=K_{\rho(\gamma) m}(f(\gamma) v, f(\gamma) w) .
$$

But $f(\gamma): T_{m} \tilde{M} \rightarrow T_{\rho(\gamma) m} \tilde{M}$ is an isomorphism, so $f(\gamma) E_{1, m}=E_{1, \rho(\gamma) m}$. Similarly, one sees that $f(\gamma) E_{2, m}=E_{2, \rho(\gamma) m}$. 
The projection of $f, \bar{f}=P f$ is a homomorphism $\pi_{1} M \rightarrow \mathrm{SO}(k)$. But $f\left(\pi_{1} M\right)$ is the holonomy of a hyperbolic structure in dimension $n$. This gives a contradiction, and we can conclude that $M$ is locally strictly convex.

A CMC foliation $\mathcal{F}$ of a space-time $V$ spatially compact Cauchy surface, is a smooth foliation $\mathcal{F}=\left\{M_{\tau}: \tau \in I\right\}$ in $V$, whose leaves are CMC Cauchy surfaces. Let $V_{\mathcal{F}}$ be the maximal domain of $V$ foliated by $\mathcal{F}$. We say that $V$ has a global CMC foliation if $V_{\mathcal{F}}=V$. As a consequence of the strict local convexity of the CMC Cauchy surface, we get a local foliation by CMC Cauchy surfaces.

Corollary 3.3. Let $V$ be a MGHF space-time, with a compact $C M C$ Cauchy surface $M$ of hyperbolic type, with mean curvature $\tau<0$. Then there is an $\varepsilon>0$, and a local $C M C$ foliation $\mathcal{F}=\left\{M_{t}: t \in(\tau-\varepsilon, \tau+\varepsilon)\right\}$.

Proof In view of the strict local convexity of $M=M_{\tau}$, the Gauss foliation w.r.t. $M_{\tau}$ can be extended both to the past and the future. Using the solution (3.1) to the Riccati equation, we see that there is an $\varepsilon>0$ so that the leaves in the Gauss foliation form barriers with $\operatorname{tr} K<\tau-\varepsilon$ to the past, and $\operatorname{tr} K>\tau+\varepsilon$ to the future. Hence by [16], we get a local foliation by CMC hypersurfaces with mean curvature taking all values in the interval $(\tau-\varepsilon, \tau+\varepsilon)$.

\subsection{Global CMC foliation.}

Next we show that the local CMC foliation can be extended to a global one. This follows from [6] in case $n=2$, and [2, Theorem 0.1] in case $n=3$. For the general case, we will use the results of [5]. The method of [2] may also be adapted to this situation, with some work.

Theorem 3.4. Let $V$ be a MGHF space-time with compact Cauchy surface $M$ of hyperbolic type. Then $V$ has a global $C M C$ foliation.

Proof Since $V$ is flat, the Einstein CMC evolution equations with zero shift, in CMC time $t=\operatorname{tr} K$, take the form

$$
\begin{aligned}
\partial_{t} g_{a b} & =-2 N K_{a b}, \\
\partial_{t} K_{a b} & =-\nabla_{a} \nabla_{b} N-N K_{a c} K_{b}^{c}, \\
-\Delta N+|K|^{2} N & =1 .
\end{aligned}
$$


Let $\mathcal{U}=(u, v), u_{a b}=g_{a b}, v_{a b}=-2 K_{a b}$, and let

$$
\begin{aligned}
L \mathcal{U} & =\left(\begin{array}{c}
\partial_{t} u-N v \\
\partial_{t} v
\end{array}\right), \\
F_{1}[u, v] & =0, \\
F_{2}[u, v] & =2 \nabla_{a} \nabla_{b} N+\frac{1}{2} N v_{a c} v_{b}^{c},
\end{aligned}
$$

where $\nabla$ is defined w.r.t. $u$, and indices are raised and lowered w.r.t. $u$. Let $F=\left(F_{1}, F_{2}\right)$. Then (3.2) is an elliptic-hyperbolic system of the form $L \mathcal{U}=$ $F$, considered in [5]. Note that $L$ is not hyperbolic in the usual sense, since the principal part is $\partial_{t}$. However, all the basic energy estimates of [5] are valid for this system, and consequently, the local existence and continuation properties hold for this system. The relevant continuation principle can be stated as follows. Let $\left(T_{-}, T_{+}\right)$be the maximal interval such that there is a CMC foliation $\mathcal{F}$ with mean curvatures taking all values in $\left(T_{-}, T_{+}\right)$. Then either $\left(T_{-}, T_{+}\right)=(-\infty, 0)$ or the quantity

$$
\max \left(\Lambda[g],|g|_{C^{1}},|N|_{C^{1}},\left|N^{-1}\right|_{L^{\infty}},|N|_{C^{1}}\right)
$$

diverges, as $t \rightarrow T_{-}$or $t \rightarrow T_{+}$. Here $\Lambda[g]$ denotes the ellipticity constant of $g$ and $|\cdot|_{C^{1}}$ denotes the $C^{1}$ norm.

Let $\tau=\operatorname{tr} K$. By the work of Treibergs [29], the estimate

$$
|K|^{2} \leq \tau^{2}
$$

holds. It follows that $|\mathrm{Ric}| \leq C_{n} \tau^{4}$. From the bound on $|K|$ we get using the maximum principle and the Lapse equation $1 / \tau^{2} \leq N \leq n / \tau^{2}$. It follows from this and the evolution equation for $g$ that the ellipticity constant and the quantities $|N|_{L^{\infty}},\left|N^{-1}\right|_{L^{\infty}}$ can't diverge as long as $\tau \in(-\infty, 0)$. It remains to consider $|g|_{C^{1}}$ and $|N|_{C^{1}}$. We will use the results of Anderson and Cheeger [4]. In view of the $|K|$ bound and the evolution equation for $g$, collapse is ruled out for $\tau \in(0, \infty)$. By the Ricci bound $|\operatorname{Ric}| \leq C_{n} \tau^{4}$, we have $g$ under control in $C^{1, \alpha}$ in harmonic coordinates and the harmonic coordinate radius $r_{h}$ is bounded away from zero. This takes care of $|g|_{C^{1}}$. In view of this regularity of $g$, and standard elliptic estimates we get from the Lapse equation that $|N|_{W^{2, p}}$ is bounded for any $p>1$, and hence $|N|_{C^{1}}$ is bounded. This proves in view of the continuation principle that the CMC foliation extends to all values of $\tau$ in $(-\infty, 0)$. In view of the Lapse bounds, it follows that proper time runs to infinity along the foliation in the expanding direction $\tau \nearrow 0$, and in view of the fact that focal distance tends to zero as $\tau \searrow-\infty$, the foliation covers all of $V$. 


\section{The limit in the expanding direction.}

In this section, we consider the limit of $M_{\tau}$ as $\tau \rightarrow 0$. We will consider the effect of scaling $V$ and $M$.

Theorem 4.1. Let $(V, \eta)$ be a MGHF space-time with Cauchy surface $M$ of hyperbolic type. Let $M_{\tau}$ be the Cauchy surface with mean curvature $\tau<0$, and let $g(\tau)$ be the induced metric on $M_{\tau}$. Then as $\tau \nearrow 0,\left(M, \frac{\tau^{2}}{n^{2}} g(\tau)\right)$ converges in $C^{0}$ to $\left(\mathbb{H}^{n}, g\right)$, where $g$ is the hyperbolic metric with sectional curvature -1 . Further the rescaled volume $\frac{|\tau|^{n}}{n^{n}} \operatorname{Vol}\left(M, g_{\tau}\right)$ tends to the hyperbolic volume, i.e.

$$
\lim _{\tau \nearrow_{0}} \frac{|\tau|^{n}}{n^{n}} \operatorname{Vol}\left(M, g_{\tau}\right) \rightarrow \operatorname{Vol}\left(M, g_{0}\right)
$$

Proof Let $U_{0} \subset H^{1}\left(\pi_{1} M, \mathbb{R}^{n+1}\right)$ be a small neighborhood of zero as in the proof of Proposition 2.3, and let $u \mapsto t(u)$ be the map from $U_{0}$ to the space of cocycles, with corresponding parametrized development map $(\gamma, u) \mapsto \mathcal{D}(\gamma, u)$, with corresponding parametrized holonomy representation $(\gamma, u) \mapsto \rho(\gamma, u)$. By the proof of Proposition 2.3, there is a $v$ such that the development map of $V$ is given by

$$
\mathcal{D}(\gamma, v) x=f(\gamma) x+t_{\gamma}(v)
$$

with cocycle $t(v)$. Then there exists $\lambda>0, u \in U_{0}$, such that $\mathcal{D}(\cdot, \lambda \cdot u)=$ $\mathcal{D}(\cdot, v)$ is the development map of $V$ with cocycle $t(v)=\lambda t(u)$.

Instead of scaling up by a large $\lambda$ as in the proof of Proposition 2.3, we will here scale down by $\lambda^{-1}$, for large $\lambda$.

We denote by $V_{\lambda}$, the space-time $V$ scaled by a factor $\lambda^{-2}$. The effect of the scaling of $V$ by $\lambda^{-2}$ can be described in two ways. First, the scaling of the cocycle is given by $t(v) \rightarrow t\left(\lambda^{-2} v\right)=\lambda^{-2} t(v)$, so as $\lambda \rightarrow \infty$, the geometric structure of $V_{\lambda}$ converges to the space-time $V_{\infty}$ with holonomy representation $f$. Secondly, the scaling can be described by $\eta \rightarrow \eta_{\lambda}=\lambda^{-2} \eta$, a scaling of the space-time metric.

Let $M_{\tau}$ be a CMC Cauchy surface of $V$ with mean curvature $\tau$, with induced metric and second fundamental form $(g, K)$. The scaling of $V$ by $\lambda^{-2}$ scales the data on $M$ by $g \rightarrow \lambda^{-2} g, K \rightarrow \lambda^{-1} K, \tau \rightarrow \lambda \tau$. Therefore, if we consider a sequence of CMC Cauchy surfaces with mean curvatures $\tau_{i}$, such that $\tau_{i} \nearrow 0$, scaling $V$ by $\tau_{i}^{2} / n^{2}$ has the effect of sending $M_{\tau_{i}}$ to a CMC Cauchy surface $M_{i}$, with mean curvature $-n$, in the space-time $V_{i}=V_{n / \tau_{i}}$. 
The sequence of space-times $V_{i}$ converges to the space-time $V_{\infty}$, as $i \rightarrow \infty$. For $i$ sufficiently large, we may apply Corollary 2.4 to find CMC hypersurfaces $N_{1, i}, N_{2, i}$ with mean curvatures $\tau_{1, i}, \tau_{2, i}$, satisfying

$$
\tau_{1, i}<-\frac{4 n}{3}+\varepsilon, \quad \tau_{2, i}>-\frac{4 n}{5}-\varepsilon,
$$

for some small $\varepsilon>0$. The hypersurfaces $N_{1, i}, N_{2, i}$ correspond to $s=$ $3 / 4,5 / 4$, respectively, in Corollary 2.4. Further, $N_{1, i}$ is in the past of $N_{2, i}$. These are therefore barriers for the CMC problem for the mean curvature $-n$. It follows by [16] that there is a CMC Cauchy surface $\bar{M}_{i}^{\prime}$ with mean curvature $-n$, such that $M_{i}^{\prime}$ is in the future of $N_{1, i}$ and in the past of $N_{2, i}$. By uniqueness of CMC hypersurfaces in space-times with compact Cauchy surface, cf. [11], [22], we see that $M_{i}^{\prime}=M_{i}$. Thus, $M_{i}$ is in the future of $N_{1, i}$ and in the past of $N_{2, i}$.

Now we pass to the universal cover. Let $\tilde{N}_{1, i}, \tilde{N}_{2, i}, \tilde{M}_{i}$ be the developements under the parametrized development map of $N_{1, i}, N_{2, i}, M_{i}$, respectively. By construction, the same causal relations hold as before. Since $V_{i}$ tends to the space-time $V_{\infty}$, as $i \rightarrow \infty$, we find that $\tilde{N}_{1, i}, \tilde{N}_{2, i}$ tend to the hyperboloids with mean curvature $-4 n / 3,-5 n / 4$, respectively, as $i \rightarrow \infty$. The convergence is uniform on compacts.

It remains to check that the volume of the induced metric tends to the volume of the hyperbolic metric on the hyperboloid $\mathbb{H}^{n} / f \pi_{1} M$. Recall that by Lemma $2.2, \tilde{M}_{i}$ is the graph of a function $\phi_{i}$ over $\mathbb{E}^{n}$. By the work of Treibergs [29, p. 54], the height function $\phi_{i}$ of $\tilde{M}_{i}$ has uniform $C^{3}$ bounds on compact subsets, and further $|D \phi|<1$ uniformly on compacts. These bounds, applied to $\tilde{M}_{i}$, together with the above barrier construction, we find that as $i \rightarrow \infty, M_{i}$ converges uniformly on compacts to the hyperboloid with mean curvature $-n$.

A calculation shows that the induced volume element $\nu_{i}$ on $\tilde{M}_{i}$ is given in terms of the gradient $\nabla \phi_{i}$ by $\nu_{i}=\sqrt{1-\left|\nabla \phi_{i}\right|^{2}} d x^{1} \cdots d x^{n}$, where $\left|\nabla \phi_{i}\right|$ is the Euclidean norm of the gradient of $\phi_{i}$. The $C^{3}$ bound shows in view of Arzela-Ascoli that $\nabla \phi_{i}$ converges uniformly on compacts, and hence the volumes $\operatorname{Vol}\left(M_{i}\right)$ satisfy

$$
\lim _{i \rightarrow \infty} \operatorname{Vol}\left(M_{i}\right)=\operatorname{Vol}\left(M, g_{0}\right) .
$$

Since the induced metric on $M_{i}$ is the rescaled metric $\frac{\tau_{i}^{2}}{n^{2}} g\left(\tau_{i}\right)$, this means precisely that the rescaled volume converges. 


\section{The Gauss map.}

Let $V$ be a MGHF space-time with compact Cauchy surface $M$ of hyperbolic type. Let $\mathbf{n}$ be the future directed time-like unit normal, $\langle\mathbf{n}, \mathbf{n}\rangle=-1$. Let as usual $\rho: \pi_{1} M \rightarrow \operatorname{ISO}(n, 1)_{0}$ be the holonomy representation of $V$ and let $f=L \rho$ be the holonomy representation of $M$.

Proposition 5.1. The map $x \mapsto \mathbf{n}(x)$ defines a well-defined map $\varphi: M \rightarrow$ $\mathbb{H}^{n} / f \pi_{1}(M)$, the Gauss map.

Proof Consider $\mathcal{D}(\tilde{M}) \subset \mathbb{R}_{1}^{n+1}$. We denote the unit future directed timelike normal of $\mathcal{D}(\tilde{M})$ by $\tilde{\mathbf{n}}$. Since $\mathbb{R}_{1}^{n+1}$ has trivial holonomy, $\tilde{\mathbf{n}}(x)$ may be identified with a point $\tilde{\varphi}(x) \in \mathbb{H}^{n}$. This defines the Gauss map $\tilde{\varphi}: \mathcal{D}(\tilde{M}) \rightarrow$ $\mathbb{H}^{n}$. In view of the fact that $\tilde{\mathbf{n}}(\rho(\gamma) x)=\rho(\gamma)_{*} \tilde{\mathbf{n}}(x)=f(\gamma) \tilde{\mathbf{n}}(x)$, the Gauss map has the following equivariance property,

$$
\tilde{\varphi}(\rho(\gamma) x)=f(\gamma) \tilde{\varphi}(x),
$$

where in the RHS, we use the action of $f \pi_{1} M$ on $\mathbb{H}^{n}$. It follows from this equivariance property, that the map $\tilde{\varphi}$ induces a map $\varphi: M \rightarrow \mathbb{H}^{n} / f \pi_{1} M$. This is the required Gauss map.

Lemma 5.1. Let $M$ be a CMC Cauchy surface in V, with Gauss map $\varphi$ : $M \rightarrow \mathbb{H}^{n} / f \pi_{1} M$. Then $\varphi$ is harmonic.

Proof Consider $\tilde{\varphi}: \mathcal{D}(\tilde{M}) \rightarrow \mathbb{H}^{n} . \mathcal{D}(\tilde{M})$ is a CMC hypersurface in $\mathbb{R}_{1}^{n+1}$ so by [12] $\tilde{\varphi}$ is harmonic. The corresponding property for $\varphi$ follows immediately.

Lemma 5.2. $\varphi$ is a diffeomorphism, which is isotopic to the identity.

Proof We may without loss of generality assume the mean curvature of $M$ is $-n$. Clearly $\varphi$ is smooth. Let $t$ be the cocycle so that $\mathcal{D}(\gamma) x=f(\gamma) x+t_{\gamma}$. By scaling the cocycle as in the proof of Proposition 2.3, we get a one parameter family of space-times and CMC hypersurfaces and Gauss maps, starting at $\mathbb{H}^{n} / f \pi_{1} M, \mathbf{I d}$ and ending at $M, \varphi$. This shows that $\varphi$ is homotopic to the identity.

Let $N$ denote $M$ with its hyperbolic metric $g_{0}$ and fix compatible orientations on $M, N$. Let $\operatorname{deg} \varphi$ denote the degree of the map $\varphi: M \rightarrow N$. Since $\operatorname{deg} \varphi$ is a homotopy invariant, we may in view of the fact that $\varphi$ is homotopic to the identity, conclude that $\operatorname{deg} \varphi=1$. 
Recall that $d \varphi: T M \rightarrow T N$ is given by the second fundamental form, $d \varphi=-K$. By Proposition $3.2,-K$ is positive definite. It follows from the inverse function theorem that $\varphi$ is locally smoothly invertible. Since $\operatorname{deg} \varphi=1$, and $d \varphi$ is positive definite, it follows that $\varphi$ is a bijection, and hence it is a diffeomorphism.

The harmonic map energy of $\varphi$, defined by $E(\varphi)=\int_{M}|d \varphi|^{2} \mu_{g}$, can be written as

$$
E(\varphi)=\int_{M}|K|^{2} d \mu_{g}=\int_{M}\left(R+(\operatorname{tr} K)^{2}\right) \mu_{g}=\int_{M} R \mu_{g}+\tau^{2} \operatorname{Vol}(M, g),
$$

where we used the constraint equation $R+(\operatorname{tr} K)^{2}=|K|^{2}$ with $R$ the scalar curvature of $(M, g)$, in the last step. In case $n=2, \int_{M} R \mu_{g}=4 \pi \chi(M)$ by Gauss-Bonnet, which gives the interesting formula

$$
E(\varphi)=4 \pi \chi(M)+\tau^{2} \operatorname{Vol}(M, g),
$$

found by Puzio [24]. In case $n \geq 3$, we note $\int_{M} R \mu_{g}<0$, in view of the fact that $M$ is of Yamabe type -1 .

\section{The rescaled volume and the $\sigma$-constant.}

Let $V$ be a MGHF space-time with Cauchy surface $M$ of hyperbolic type, and let $\mathcal{F}=\left\{M_{\tau}: \tau \in(-\infty, 0)\right\}$ be the global CMC foliation of $V$ as in Theorem 3.4. In the rest of this section, $M$ will denote a CMC Cauchy surface, with induced metric $g$ and second fundamental form $K$, with $\operatorname{tr} K=$ $\tau$, constant on $M$. Let

$$
\mathcal{H}(\tau)=\mathcal{H}(g, K)=|\tau|^{n} \operatorname{Vol}(M, g)=|\tau|^{n} \int_{M} \mu_{g}
$$

Then $\mathcal{H}(g, K)$ can be thought of as a function on the space of CMC flat data sets on $M$.

The infimum $\inf _{g, K} \mathcal{H}(g, K)$ of $\mathcal{H}$ over all CMC flat data on $M$, is a quantity that is analogous to the $\sigma$-constant of $M$, but in the restricted setting of flat CMC data.

Via the Yamabe equation, we may write the metric $g$ as $g=u^{\frac{4}{n-2}} h$, where $h$ has constant negative scalar curvature $-n(n-1)$. Let

$$
K=\frac{\tau}{n} g+u^{-2} \sigma
$$


where $\sigma$ is satisfies $\operatorname{tr}_{h} \sigma=0, \nabla^{a}[h] \sigma_{a b}=0$. Then $K$ is divergence free w.r.t. $g$, and using the Yamabe equation, the vacuum constraint equation $R_{g}+\left(\operatorname{tr}_{g} K\right)^{2}-|K|_{g}^{2}=0$, takes the form

$$
-\frac{4(n-1)}{n-2} \Delta_{h} u+R_{h} u+\frac{n-1}{n} \tau^{2} u^{\frac{n+2}{n-2}}-u^{\frac{2-3 n}{n-2}}|\sigma|_{h}^{2}
$$

Thus we may write

$$
\mathcal{H}(g, K)=|\tau|^{n} \int_{M}\left(u^{\frac{4}{n-2}}\right)^{n / 2} \mu_{h}
$$

where via the constraint equation, we have $u=u(h, \sigma, \tau)$. A maximum principle argument shows that $u(h, \sigma, \tau) \geq u(h, 0, \tau)$, and hence we get that $u(h, 0, \tau)$ satisfies

$$
u^{\frac{4}{n-2}}=\frac{n^{2}}{\tau^{2}}
$$

This gives

$$
\mathcal{H} \geq n^{n} \operatorname{Vol}(M, h)
$$

where $h$ is the unique metric in the conformal class of $g$ with scalar curvature $R_{h}=-n(n-1)$ and shows that

$$
\inf \mathcal{H}(g, K)=n^{n} \inf _{h \in \mathcal{M}_{-n(n-1)}} \operatorname{Vol}(M, h)
$$

where the infimum is over the space of CMC vacuum data on $M$. It follows that

$$
\sigma(M)=-\frac{n-1}{n} \inf \mathcal{H}^{2 / n}
$$

These relations have been studied in case $n=3$ by Fischer and Moncrief [14].

Theorem 6.1. Let $M$ be a compact manifold of hyperbolic type, of dimension $n \geq 3$, and let $g_{0}$ be the hyperbolic metric on $M$ with sectional curvature -1 .

Let $(g, K)$ be flat data set on $M$ with mean curvature $\tau=\operatorname{tr} K$. Then the rescaled volume $\mathcal{H}(g, K)=|\tau|^{n} \operatorname{Vol}(M, g)$ satisfies

$$
\mathcal{H}(g, K) \geq n^{n} \operatorname{Vol}\left(M, g_{0}\right) .
$$

Equality in (6.1) holds if and only if $g$ is isometric to $\frac{n^{2}}{\tau^{2}} g_{0}$ and $K=\frac{\operatorname{tr} K}{n} g$. 
Proof We will use a monitonicity property for $\mathcal{H}$, which was first noticed by A. Rendall (unpublished) and studied in detail by Fisher and Moncrief [14] for dimension $n=3$.

Let $\hat{K}$ denote the tracefree part of $K, K=\frac{\tau}{n} g+\hat{K}$. By integrating the Lapse equation we see

$$
\int_{M}\left(\frac{d \tau}{d t}-N \frac{\tau^{2}}{n}\right) \mu_{g}=\int_{M} N|\hat{K}|^{2} \mu_{g}
$$

Time differentiation $\mathcal{H}(\tau)$ using the evolution equation (3.2a) and shows

$$
\partial_{t} \mathcal{H}(\tau)=-n|\tau|^{n-1} \int_{M} N|\hat{K}|^{2} \mu_{g} \leq 0
$$

and $\partial_{t} H(\tau)=0$ if and only if $\hat{K}=0$.

In case $\hat{K}=0$ at $\tau$, a calculation shows $\partial_{t}^{2} \mathcal{H}(\tau)=0$. Further, in this case,

$$
\partial_{t}^{3} H(\tau)=-|\tau|^{n-1} n \int N\left|\partial_{t} \hat{K}\right|^{2} \mu_{g}
$$

In case $\hat{K}=0$ at $\tau, N=n / \tau^{2}$ by the Lapse equation with $d \tau / d t=1$ and the evolution equations (3.2), with we get

$$
\partial_{t} \hat{K}_{a b}=\frac{2}{n} g
$$

This shows that in case $\partial_{t} \mathcal{H}(\tau)=0$, then $\partial_{t}^{2} \mathcal{H}(\tau)=0$, and

$$
\partial_{3} \mathcal{H}(\tau)=-4 n|\tau|^{n-3} \operatorname{Vol}(M, g)<0
$$

This shows that $\mathcal{H}$ is monotonically decreasing. As $\mathcal{H}$ is nonnegative, it follows that the $\operatorname{limit}_{\lim _{\tau} \nearrow_{0}} \mathcal{H}(\tau)$ exists, and

$$
\lim _{\tau \nearrow 0} \mathcal{H}(\tau)=\inf _{\tau \in(-\infty, 0)} \mathcal{H}(\tau)
$$

On the other hand, by Theorem 4.1, $\lim _{\tau} \nearrow_{0} \mathcal{H}(\tau)=n^{n} \operatorname{Vol}\left(M, g_{0}\right)$, where $g_{0}$ is the hyperbolic metric on $M$ with sectional curvature -1 . This proves (6.1).

It remains to prove the rigidity statement. Suppose that $(g, K)$ are flat CMC data such that $\mathcal{H}(\tau)=\mathcal{H}(g, K)=n^{n} \operatorname{Vol}\left(M, g_{0}\right)$. Then $\partial_{t} \mathcal{H}(\tau)=0$, which implies that $K=\frac{\operatorname{tr} K}{n} g$. After scaling, we may assume $\operatorname{tr} K=-n$. It follows that

$$
R_{a b}=-(n-1) g_{a b}
$$


In case $n=3$ this is enough to complete the proof. For the higher dimensional case, however, it follows from $\mathcal{H}(g, K)=n^{n} \operatorname{Vol}\left(M, g_{0}\right)$ that $\operatorname{Vol}(M, g)=\operatorname{Vol}\left(M, g_{0}\right)$, and therefore, by [9], $(M, g)$ is isometric to $\left(M, g_{0}\right)$. This completes the proof.

\section{References.}

[1] M. T. Anderson, Scalar curvature, metric degenerations and the static vacuum Einstein equations on 3-manifolds. I, Geom. Funct. Anal. 9 (1999), no. 5, 855-967.

[2] M. T. Anderson, On long-time evolution in general relativity and geometrization of 3-manifolds, 2000, preprint, Stony Brook.

[3] Michael T. Anderson, Scalar curvature and geometrization conjectures for 3-manifolds, Comparison geometry (Berkeley, CA, 1993-94), Cambridge Univ. Press, Cambridge, 1997, pp. 49-82.

[4] Michael T. Anderson and Jeff Cheeger, Diffeomorphism finiteness for manifolds with Ricci curvature and $L^{n / 2}$-norm of curvature bounded, Geom. Funct. Anal. 1 (1991), no. 3, 231-252.

[5] Lars Andersson and Vincent Moncrief, Elliptic-hyperbolic systems and the Einstein equations, submitted to Ann. Henri Poincaré.

[6] Lars Andersson, Vincent Moncrief, and Anthony J. Tromba, On the global evolution problem in $2+1$ gravity, J. Geom. Phys. 23 (1997), no. 3-4, 191-205.

[7] M. Artin, On the solutions of analytic equations, Invent. Math. 5 (1968), 277-291.

[8] G. Besson, G. Courtois, and S. Gallot, Volume et entropie minimale des espaces localement symétriques, Invent. Math. 103 (1991), no. 2, 417-445.

[9] , Entropies et rigidités des espaces localement symétriques de courbure strictement négative, Geom. Funct. Anal. 5 (1995), no. 5, 731-799.

[10] I. Bivens, J.-P. Bourguignon, A. Derdziński, D. Ferus, O. Kowalski, T. Klotz Milnor, V. Oliker, U. Simon, W. Strübing, and K. Voss, Discussion on Codazzi-tensors, Global differential geometry and global analysis (Berlin, 1979), Springer, Berlin, 1981, pp. 243-299. 
[11] Dieter Brill and Frank Flaherty, Isolated maximal surfaces in spacetime, Comm. Math. Phys. 50 (1976), no. 2, 157-165.

[12] Hyeong In Choi and Andrejs Treibergs, Gauss maps of spacelike constant mean curvature hypersurfaces of Minkowski space, J. Differential Geom. 32 (1990), no. 3, 775-817.

[13] Yvonne Choquet-Bruhat and Robert Geroch, Global aspects of the Cauchy problem in general relativity, Comm. Math. Phys. 14 (1969), 329-335.

[14] Arthur E. Fischer and Vincent Moncrief, The Einstein flow, the $\sigma$ constant and the geometrization of 3-manifolds, Classical Quantum Gravity 16 (1999), no. 11, L79-L87.

[15] S. Gallot, Curvature-decreasing maps are volume-decreasing (on joint work with G. Besson and G. Courtois), Proceedings of the International Congress of Mathematicians, Vol. II (Berlin, 1998), vol. 1998, pp. 339348 (electronic).

[16] Claus Gerhardt, $H$-surfaces in Lorentzian manifolds, Comm. Math. Phys. 89 (1983), no. 4, 523-553.

[17] William M. Goldman and John J. Millson, Deformations of flat bundles over Kähler manifolds, Geometry and topology (Athens, Ga., 1985), Dekker, New York, 1987, pp. 129-145.

[18] Dennis Johnson and John J. Millson, Deformation spaces associated to compact hyperbolic manifolds, Discrete groups in geometry and analysis (New Haven, Conn., 1984), Birkhäuser Boston, Boston, Mass., 1987, pp. 48-106.

[19] Michael Kapovich, Deformations of representations of discrete subgroups of so(3,1), Math. Ann. 299 (1994), no. 2, 341-354.

[20] Jacques Lafontaine, Modules de structures conformes plates et cohomologie de groupes discrets, C. R. Acad. Sci. Paris Sér. I Math. 297 (1983), no. 13, 655-658.

[21] Claude LeBrun, Kodaira dimension and the Yamabe problem, Comm. Anal. Geom. 7 (1999), no. 1, 133-156.

[22] Jerrold E. Marsden and Frank J. Tipler, Maximal hypersurfaces and foliations of constant mean curvature in general relativity, Phys. Rep. 66 (1980), no. 3, 109-139. 
[23] G. Mess, Lorentz spacetimes of constant curvature, Tech. Report IHES/M/90/28, Institute des Hautes Etudes Scientifiques, 1990.

[24] Raymond S. Puzio, The Gauss map and 2+1 gravity, Classical Quantum Gravity 11 (1994), no. 11, 2667-2675.

[25] M. S. Raghunathan, Discrete subgroups of Lie groups, Springer-Verlag, New York, 1972, Ergebnisse der Mathematik und ihrer Grenzgebiete, Band 68.

[26] John G. Ratcliffe, Foundations of hyperbolic manifolds, Springer-Verlag, New York, 1994.

[27] Kevin P. Scannell, 3-manifolds which are spacelike slices of flat spacetimes, 1999.

$[28]$ 2000, private communication.

[29] Andrejs E. Treibergs, Entire spacelike hypersurfaces of constant mean curvature in Minkowski space, Invent. Math. 66 (1982), no. 1, 39-56.

DePaRTMENT OF MATHEMATiCS

UNIVERSITY OF MIAMI

Coral Gables, FL 33124

USA

E-mail: larsa@math.miami.edu 